Pis'ma v ZhETF

\title{
Real-Time Instantons and Suppression of Collision-Induced Tunneling
}

\author{
D. Levkov ${ }^{a, b 1)}$, S. Sibiryakov ${ }^{a}$ \\ ${ }^{a}$ Institute for Nuclear Research of the Russian Academy of Sciences, \\ 60th October Anniversary prospect 7a, Moscow 117312, Russia \\ ${ }^{b}$ Moscow State University, Department of Physics, Vorobjevy Gory, Moscow, 119899, Russia
}

\begin{abstract}
We consider tunneling processes in QFT induced by collisions of elementary particles. We propose a semiclassical method for estimating the probability of these processes in the limit of very high collision energy. As an illustration, we evaluate the maximum probability of induced tunneling between different vacua in a $(1+1)$-dimensional scalar model with boundary interaction.
\end{abstract}

PACS: $11.15 \mathrm{Kc}, 03.70+\mathrm{k}, 03.65 \mathrm{Sq}$

In many models of field theory one encounters tunneling transitions between states separated by an energy barrier of finite height $E_{S}$, the famous examples are false vacuum decay in scalar theories [1] and topologychanging transitions in gauge-Higgs theory 2] 3. In the weak coupling regime, the rate of tunneling at zero energy is exponentially small [1, 2], but one suspects the suppression to vanish once energy exceeding the height of the barrier is injected into the system. A particular way of inducing the tunneling process is a collision of two highly energetic particles. It was conjectured some time ago [4] that the collision-induced tunneling processes may become unsuppressed at high collision energies. Semiclassical study of scalar and gauge-Higgs theories [5] 6] showed, however, that this is not the case: the tunneling probability remains exponentially small even if the collision energy $E$ exceeds considerably the barrier height $E_{S}$. Furthermore, analyses of toy models [7, 8, and unitarity arguments [9] suggest that the collision-induced transitions should remain exponentially suppressed even if $E$ tends to infinity. In other words, it was proposed that, contrary to the initial conjecture, the probability of the process has the form,

$$
\mathcal{P}(E) \propto \mathrm{e}^{-F(E) / g^{2}},
$$

where $g^{2}$ is a small coupling constant, and the suppression exponent $F(E)$ remains positive at all energies. This has been confirmed recently by direct calculation [10] of the suppression exponent in the whole range of energies in a toy two-dimensional model. In addition, it was found in [10] that the suppression exponent reaches its minimum $F_{\mathrm{m}}$ at certain optimal energy $E_{\mathrm{o}}$ and remains constant above this energy,

$$
F(E)=F_{\mathrm{m}}, \quad E>E_{\mathrm{o}} .
$$

\footnotetext{
1)e-mail: levkov@ms2.inr.ac.ru
}

One may ask whether or not the formulas (11), (21) are valid for other models, with model-dependent values of $F_{\mathrm{m}}, E_{\mathrm{o}}$.

In this letter we give a general semiclassical method for evaluating the minimum value $F_{\mathrm{m}}$ of the suppression exponent and the energy $E_{\mathrm{o}}$ at which this minimum is achieved. Our procedure is essentially an adaptation of the method of Ref. 11], but it is more straightforward and technically simpler. We consider the case in which, after the appropriate rescaling of the fields, the action $S$ takes the form $S=\tilde{S} / g^{2}$, where $\tilde{S}$ does not explicitly depend on the small coupling constant $g$. One observes that $g^{2}$ plays effectively the role of the Planck constant, and the limit $g \rightarrow 0$ which we consider below, corresponds to a semiclassical situation.

Our starting point is the inclusive probability of tunneling from states with a given number of incoming particles and any energy,

$$
\mathcal{P}_{\mathrm{m}}(N)=\sum_{i, f}\left|\left\langle f\left|\hat{\mathcal{U}}\left(T_{f}, T_{i}\right) \hat{P}_{N}\right| i\right\rangle\right|^{2} .
$$

Here $\hat{\mathcal{U}}\left(T_{f}, T_{i}\right)$ is the evolution operator, and $\hat{P}_{N}$ denotes the projector onto states with $N$ particles. The initial and final states, $|i\rangle$ and $|f\rangle$, respectively, are at the different sides of the potential barrier. Here and below the limit $T_{f} \rightarrow+\infty, T_{i} \rightarrow-\infty$ is assumed. As we will shortly see, the quantity (3) can be evaluated semiclassically provided the initial number of particles is parametrically large, $N=\tilde{N} / g^{2}$. We will see that the result has a typical exponential form,

$$
\mathcal{P}_{\mathrm{m}}(N) \propto \mathrm{e}^{-F_{\mathrm{m}}(\tilde{N}) / g^{2}} .
$$

We use the result (4) as a source of information on the probability of collision-induced tunneling. It is clear that the inclusive multiparticle probability $\mathcal{P}_{\mathrm{m}}(N)$ sets an upper bound on the two-particle probability of interest, $\mathcal{P}(E)$, at arbitrarily high energies $E$. Indeed, the 
energy of the initial state in (3) can be arbitrarily high, while any initial two-particle state can be promoted to the multiparticle one by adding a number of "spectator" particles which do not interfere with the tunneling process. Hence, the exponential suppression of $\mathcal{P}_{\mathrm{m}}(N)$ entails the exponential suppression of $\mathcal{P}(E)$, and the inequality

$$
F_{\mathrm{m}}(\tilde{N}) \leq F_{\mathrm{m}}
$$

holds. Following Ref. [11, we conjecture that

$$
\lim _{\tilde{N} \rightarrow 0} F_{\mathrm{m}}(\tilde{N})=F_{\mathrm{m}} .
$$

The conjecture (6) is based on the observation that in the leading semiclassical approximation the probability of tunneling does not depend on the details of the initial state provided the initial number of particles is much smaller than $1 / g^{2}$. The analogs of the formula (6) have been checked in various situations [12, 13, 14.

Now, we proceed to the semiclassical evaluation of the multiparticle exponent $F_{\mathrm{m}}(\tilde{N})$. Our strategy is to represent the inclusive probability $\mathcal{P}_{\mathrm{m}}(N)$ in the form of path integral and evaluate the latter making use of the saddle-point technique. Taking into account that $\hat{P}_{N} \cdot \hat{P}_{N}=\hat{P}_{N}$, one recasts Eq. (3) in the form,

$$
\begin{aligned}
& \mathcal{P}_{\mathrm{m}}(N)= \sum_{i^{\prime}, i^{\prime \prime}, f}\left\langle f|\hat{\mathcal{U}}| i^{\prime}\right\rangle\left\langle i^{\prime}\left|\hat{P}_{N}\right| i^{\prime \prime}\right\rangle\left\langle i^{\prime \prime}|\hat{\mathcal{U}}| f\right\rangle \\
&=\int \mathcal{D}\left[\phi_{f}, a, a^{*}, b, b^{*}\right] \mathrm{e}^{-\frac{1}{g^{2}} \int d \mathbf{k}\left[a_{\mathbf{k}} a_{\mathbf{k}}^{*}+b_{\mathbf{k}} b_{\mathbf{k}}^{*}\right]} \\
& \quad \times\left\langle\phi_{f}|\hat{\mathcal{U}}| a\right\rangle\left\langle a\left|\hat{P}_{N}\right| b\right\rangle\left[\left\langle\phi_{f}|\hat{\mathcal{U}}| b\right\rangle\right]^{*}
\end{aligned}
$$

where $\left|\phi_{f}\right\rangle$ are eigenstates of the field operators which we denote collectively by $\hat{\phi}$, while $|a\rangle,|b\rangle$ are the coherent states,

$$
\hat{\phi}(\mathbf{x})|\phi\rangle=\frac{\phi(\mathbf{x})}{g}|\phi\rangle, \quad \hat{a}_{\mathbf{k}}|a\rangle=\frac{a_{\mathbf{k}}}{g}|a\rangle .
$$

Hereafter we use the shorthand notation $\hat{\mathcal{U}} \equiv \hat{\mathcal{U}}\left(T_{f}, T_{i}\right)$. Making use of the standard path integral for the transition amplitude in $\phi$-representation, one writes

$$
\left\langle\phi_{f}|\hat{\mathcal{U}}| a\right\rangle=\int_{\phi\left(T_{f}\right)=\phi_{f}} \mathcal{D} \phi \exp \left\{\frac{1}{g^{2}}\left(i \tilde{S}[\phi]+B_{i}\left(\phi_{i}, a\right)\right)\right\},
$$

where the boundary term $B_{i}$ comes from the initial matrix element $\langle\phi \mid a\rangle$,

$$
\begin{aligned}
B_{i}\left(\phi_{i}, a\right)=\int & d \mathbf{k}\left\{-\frac{1}{2} a_{\mathbf{k}} a_{-\mathbf{k}}\right. \\
& \left.-\frac{\omega_{\mathbf{k}}}{2} \phi_{i}(\mathbf{k}) \phi_{i}(-\mathbf{k})+\sqrt{2 \omega_{\mathbf{k}}} a_{\mathbf{k}} \phi_{i}(\mathbf{k})\right\} .
\end{aligned}
$$

In this expression $\phi_{i}(\mathbf{k})$ stands for the spatial Fourier transform of the fields at $t=T_{i}$. For the matrix element of the projector $\hat{P}_{N}$ one obtains (see e.g. [15] 16])

$$
\left\langle a\left|\hat{P}_{N}\right| b\right\rangle=\int_{-i \infty}^{i \infty} d \theta \exp \left\{\frac{1}{g^{2}}\left(\tilde{N} \theta+\int d \mathbf{k} a_{\mathbf{k}}^{*} b_{\mathbf{k}} \mathrm{e}^{-\theta}\right)\right\} .
$$

Substituting Eqs. (8), (9) into the expression (7) and performing integration over the variables $b, b^{*}$, one obtains the desired path integral representation,

$$
\mathcal{P}_{\mathrm{m}}(N)=\int_{\phi\left(T_{f}\right)=\phi^{\prime}\left(T_{f}\right)} \mathcal{D}\left[\phi, \phi^{\prime}, a, a^{*}\right] d \theta \mathrm{e}^{-F / g^{2}},
$$

where

$$
\begin{aligned}
F=-\tilde{N} \theta-i \tilde{S}[\phi]+i \tilde{S}\left[\phi^{\prime}\right] \\
\quad-B_{i}\left(\phi_{i}, a\right)-B_{i}^{*}\left(\phi_{i}^{\prime}, a\right)+\int d \mathbf{k} a_{\mathbf{k}}^{*} a_{\mathbf{k}} \mathrm{e}^{\theta} .
\end{aligned}
$$

Note that the integration over $\phi^{\prime}$ in (10) comes from the path integral representation for the complex conjugate amplitude $\left[\left\langle\phi_{f}|\hat{\mathcal{U}}| b\right\rangle\right]^{*}$.

The functional $F$ defined in Eq. (11) is independent of the coupling constant $g$. Hence, at weak coupling the integral (10) is saturated by its saddle point. The saddle-point equations are as follows. Extremization with respect to $\phi$ and $\phi^{\prime}$ gives the classical field equations

$$
\frac{\delta \tilde{S}}{\delta \phi}=\frac{\delta \tilde{S}}{\delta \phi^{\prime}}=0 .
$$

Boundary conditions for these equations are obtained by varying the expression (11) with respect to the initial and final values of the fields. Using the relation $\delta S / \delta \phi\left(T_{f}, \mathbf{x}\right)=\dot{\phi}\left(T_{f}, \mathbf{x}\right)$ and taking into account the constraint (see. Eq.(10)

$$
\phi\left(T_{f}, \mathbf{x}\right)=\phi^{\prime}\left(T_{f}, \mathbf{x}\right),
$$

one obtains,

$$
\dot{\phi}\left(T_{f}, \mathbf{x}\right)=\dot{\phi}^{\prime}\left(T_{f}, \mathbf{x}\right) .
$$

In the initial asymptotic region $T_{i} \rightarrow-\infty$ the evolution of the fields $\phi, \phi^{\prime}$ is linear, and one writes ${ }^{2)}$

$$
\phi_{i}=\int \frac{d \mathbf{k}}{(2 \pi)^{1 / 2} \sqrt{2 \omega_{\mathbf{k}}}}\left(f_{\mathbf{k}} \mathrm{e}^{-i \omega_{\mathbf{k}} T_{i}}+g_{-\mathbf{k}}^{*} \mathrm{e}^{i \omega_{\mathbf{k}} T_{i}}\right) \mathrm{e}^{i \mathbf{k x}},
$$

\footnotetext{
${ }^{2)}$ For concreteness we assume that the initial state is an excitation above the vacuum $\phi=0$.
} 


$$
\phi_{i}^{\prime}=\int \frac{d \mathbf{k}}{(2 \pi)^{1 / 2} \sqrt{2 \omega_{\mathbf{k}}}}\left(f_{\mathbf{k}}^{\prime} \mathrm{e}^{-i \omega_{\mathbf{k}} T_{i}}+g_{-\mathbf{k}}^{\prime *} \mathrm{e}^{i \omega_{\mathbf{k}} T_{i}}\right) \mathrm{e}^{i \mathbf{k x}} .
$$

The variation of the functional $F$ with respect to $\phi_{i}$, $\phi_{i}^{\prime}, a, a^{*}$ yields the following relations between the frequency components,

$$
f_{\mathbf{k}}^{\prime}=f_{\mathbf{k}} \mathrm{e}^{\theta}, \quad g_{\mathbf{k}}^{\prime *}=g_{\mathbf{k}}^{*} \mathrm{e}^{-\theta} .
$$

The set of saddle-point equations (12) can be simplified if we recall that the configurations $\phi$ and $\phi^{\prime}$ saturate the amplitude and its complex conjugate, respectively. This suggests the Ansatz $\phi^{\prime}(t, \mathbf{x})=[\phi(t, \mathbf{x})]^{*}$, which is compatible with the boundary value problem (12) provided the saddle-point value of $\theta$ is real. Then, the boundary value problem is formulated in terms of a single set of fields $\phi(t, \mathbf{x})$. The conditions (12b), (12c) imply the reality of the fields in the asymptotic future,

$$
\operatorname{Im} \phi(t, \mathbf{x}) \rightarrow 0, \operatorname{Im} \dot{\phi}(t, \mathbf{x}) \rightarrow 0 \text { as } t \rightarrow+\infty,
$$

while Eqs. (12d) read

$$
f_{\mathbf{k}}=\mathrm{e}^{-\theta} g_{\mathbf{k}} .
$$

The boundary condition in the asymptotic past, Eq. 13b), can be understood as follows. In the limit $\theta \rightarrow+\infty$ it coincides with the Feynman boundary condition and thus corresponds to the initial state with semiclassically small number of particles, $\tilde{N} \rightarrow 0$; finite $\theta$ picks up the most favourable state with non-zero $\tilde{N}$.

The number of equations in the boundary value problem (12a), (13) is equal to the number of unknowns. Generically, for a given value of $\theta$ this problem has a unique solution $\phi_{r t}(t, \mathbf{x})$. We call this solution "realtime instanton", as it lives on the real time axis, in contrast to the ordinary instanton which is defined in Euclidean time. Note that the boundary condition (13b) implies that the real-time instanton is complexvalued. On the other hand, its imaginary part should vanish in the asymptotic future due to the condition (13a). Let us discuss the consequences of this property. Assume that at large (but finite) times the solution gets linearized about some real static configuration, $\phi_{r t}(t, \mathbf{x})=\phi_{s}(\mathbf{x})+\delta \phi(t, \mathbf{x})$. Then Eq.(12a) implies that $\operatorname{Im} \delta \phi \rightarrow 0$ as $t \rightarrow+\infty$. This amounts to requiring that the configuration $\phi_{s}$ is unstable, so that $\operatorname{Im} \delta \phi$ evolves along its negative mode, $\operatorname{Im} \delta \phi \propto \mathrm{e}^{- \text {const.t. The natural }}$ candidate for $\phi_{s}(\mathbf{x})$ is the static solution "sitting" on top of the potential barrier separating the sectors of initial and final states. Accepting the terminology of gaugeHiggs theories [3] we call this solution "sphaleron"3).

\footnotetext{
${ }^{3)}$ In case of scalar theories such solution is known as "critical bubble" [1].
}

We arrive at the conclusion that the real-time instantons describe formation of the sphaleron as $t \rightarrow+\infty$. This is in fact a common property of solutions relevant for collision-induced tunneling at energies higher than $E_{S}$ [14, 6, 10. The transition is completed by the decay of the sphaleron into the states of interest, which proceeds with probability of order one.

The last saddle-point equation obtained by varying the functional (11) with respect to the parameter $\theta$, relates the value of $\theta$ to the initial number of particles,

$$
\tilde{N}=\int d \mathbf{k} f_{\mathbf{k}} g_{\mathbf{k}}^{*} .
$$

Substituting the solution into Eq. (11), one obtains the formula (4), with the suppression exponent

$$
F_{\mathrm{m}}(\tilde{N})=-\tilde{N} \theta+2 \operatorname{Im}\left\{\tilde{S}\left[\phi_{r t}\right]+\left.\frac{1}{2} \int d \mathbf{x} \phi_{r t} \dot{\phi}_{r t}\right|_{t=T_{i}}\right\} .
$$

Note that the term in braces is the action of the realtime instanton integrated by parts with respect to time.

At finite value of the parameter $\theta$ (non-zero value of $\tilde{N}$ ) the real-time instanton is, by construction, a smooth solution to the equations of motion and has a well-defined classical energy $E_{\mathrm{o}}(\tilde{N})$. At $t \rightarrow+\infty$ the real-time instanton describes semiclassical evolution in the final state. Thus, $E_{\mathrm{o}}(\tilde{N})$ coincides with the energy of the final state saturating the probability (3). This means that if we restrict the sum in (3) to the states with the fixed energy $E=E_{\mathrm{o}}(\tilde{N})$ (cf. Ref. 111) we obtain the same result (4). One concludes that $E_{\mathrm{o}}(\tilde{N})$ is the optimal energy for tunneling from the states with given number of particles $N=\tilde{N} / g^{2}$. The limit

$$
E_{\mathrm{O}}=\lim _{\tilde{N} \rightarrow 0} E_{\mathrm{o}}(\tilde{N})
$$

determines the optimal energy for collision-induced tunneling ${ }^{4)}$. It is easy to understand the most favourable transition at higher energies. The system releases the energy excess $\left(E-E_{\mathrm{o}}\right)$ by a perturbative emission of a few particles (which costs only a power suppression in $\left.g^{2}\right)$ and tunnels at the optimal energy $E_{\mathrm{o}}$ [7, 10]. Due to this process $F(E)$ stays constant at $E>E_{\mathrm{o}}$, i.e. the formula (2) holds.

Let us illustrate our method by considering a simple example. We consider free massless scalar field $\phi(t, x)$ living in $(1+1)$ dimensions on a half-line $x>0$, with

\footnotetext{
${ }^{4)}$ In general, we cannot exclude the situation when $E_{\mathrm{o}}(\tilde{N}) \rightarrow \infty$ as $\tilde{N} \rightarrow 0$. In the example below, $E_{\text {o }}$ is finite.
} 
self-interaction localized at the boundary point $x=0$. The action of the model is ${ }^{5)}$

$$
S=\frac{1}{g^{2}} \int d t\left\{\frac{1}{2} \int_{0}^{\infty} d x\left(\partial_{\mu} \phi\right)^{2}-\mu[1-\cos (\phi(t, 0))]\right\},
$$

where the parameter $\mu$ sets the characteristic energy scale of the boundary interaction. The model (17) is used in solid state physics to describe the transport in quantum wires [17] and Josephson chains with defects [18. A detailed semiclassical treatment of the model (17) is given in [10.

The model (17) has a number of vacua $\phi_{n}=2 \pi n$, $n=0, \pm 1, \ldots$, which are separated by the potential barriers of the height $E_{S}=2 \mu / g^{2}$ determined by the maximum of the boundary potential. One also finds an unstable static solution $\phi_{s}=\pi$, sphaleron, which "sits" on the top of the "first" potential barrier. The process we are interested in is tunneling between the vacua $\phi=0$ and $\phi=2 \pi$ induced by a highly energetic particle scattering off the boundary. To calculate the minimum suppression exponent and the optimal energy of this collision-induced tunneling process, one finds the family of real-time instantons by solving the boundary value problem (12a), (13).

Since the bulk evolution of $\phi(t, x)$ is that of free massless scalar field, we represent the general solution in the form

$$
\phi(t, x)=\varphi_{\text {in }}(t+x)+\varphi_{\text {out }}(t-x),
$$

where $\varphi_{\text {in }}$ and $\varphi_{\text {out }}$ are the incoming and outgoing waves. The boundary interaction leads to non-linear equation at $x=0$,

$$
\partial_{x} \phi=\mu \sin \phi, \quad x=0 .
$$

Due to the condition (13a), the outgoing wave $\varphi_{\text {out }}$ is real. Introducing the real and imaginary parts of the incoming wave, $\varphi_{\text {in }}(\xi)=a(\xi)+i b(\xi)$, one rewrites Eq. (19) as a set of two real equations,

$$
\begin{aligned}
& b^{\prime}=\mu \operatorname{sh} b \cos u, \\
& u^{\prime}=2 a^{\prime}-\mu \operatorname{ch} b \sin u,
\end{aligned}
$$

where $u(\xi)=a(\xi)+\varphi_{\text {out }}(\xi)$. The remaining conditions in the asymptotic past, Eq. (13b), should be imposed

\footnotetext{
5) Normally, one should use some infrared regularization for the massless scalar model in $(1+1)$ dimensions. However, the specifics of regularization turn out to be irrellevant for our purposes.
}

on the frequency components of the incoming wave $\varphi_{\text {in }}$. To this end, one performs Fourier expansion of $\varphi_{\mathrm{in}}$,

$$
\varphi_{\text {in }}(t+x)=\int d k \varphi_{\text {in }}(k) \mathrm{e}^{i k(t+x)},
$$

and finds that the positive and negative frequency components of the solution, $f_{-k}$ and $g_{-k}^{*}$, are proportional to $\varphi_{\text {in }}(-k)$ and $\varphi_{\text {in }}(k), k>0$, respectively. Thus, Eq. (13b) takes the form $\varphi_{\text {in }}(-k)=\mathrm{e}^{-\theta}\left[\varphi_{\text {in }}(k)\right]^{*}$, where $k>0$. It is straigforward to check using the Cauchy formula that the latter condition is equivalent in its turn to the following relation between the real and imaginary parts of the initial wave (see Ref. [10]),

$$
a^{\prime}(\xi)=\frac{1+\mathrm{e}^{-\theta}}{1-\mathrm{e}^{-\theta}} \cdot \frac{1}{\pi \xi} \text { V.P. } \int d \xi_{1} \frac{\xi_{1} b^{\prime}\left(\xi_{1}\right)}{\xi_{1}-\xi},
$$

where the integral is understood in the sense of principal value. We use Eq. (23) as an alternative formulation of the condition (13b) in our model.

One also requires that the functions $\varphi_{\text {in }}, \varphi_{\text {out }}$ have appropriate asymptotics. To ensure the finite number of particles in the initial state we require the incoming wave to be well localized, $\varphi_{\text {in }}(\xi) \rightarrow 0$ as $\xi \rightarrow \pm \infty$. Besides, as the initial state is an excitation above the vacuum $\phi=0$, we write $\varphi_{\text {out }}(\xi) \rightarrow 0$ at $\xi \rightarrow-\infty$. On the other hand, as we have already discussed, the relevant solution contains the sphaleron at $t \rightarrow+\infty$. Thus, $\varphi_{\text {out }}(\xi) \rightarrow \pi$ as $\xi \rightarrow+\infty$.

The problem (20), (21), 231) can be solved numerically by the following iterative method. At each cycle of iterations one starts from the function ${ }^{6)} u=u^{(0)}(\xi)$ and solves Eq. (20) explicitly,

$$
b(\xi)=\ln \operatorname{th}\left(-\frac{\mu}{2} \int_{0}^{\xi} \cos u^{(0)}\left(\xi_{1}\right) d \xi_{1}+\varkappa\right),
$$

where $\varkappa$ is an integration constant. Then, integrating numerically Eqs. (23), (21), one finds $a(\xi)$ and $u(\xi)$ for given value of $\varkappa$. Finally, one picks up the value of $\varkappa$ such that the function $u(\xi)$ has correct asymptotics as $\xi \rightarrow \pm \infty$. In this way the improved approximation for $u(\xi)$ is obtained, and a new cycle of iterations begins. After 30 cycles one obtains the solution with precision of order $10^{-6}$.

Given the family of real-time instantons, one calculates numerically the suppression exponent $F_{\mathrm{m}}(\theta)$ via Eq. (15), and also the energy $E_{\mathrm{o}}(\theta)$. To calculate the

\footnotetext{
${ }^{6)}$ At the very first cycle one takes, e.g., $u^{(0)}(\xi)=\pi / 2+$ $\arctan (\mu \xi)$.
} 

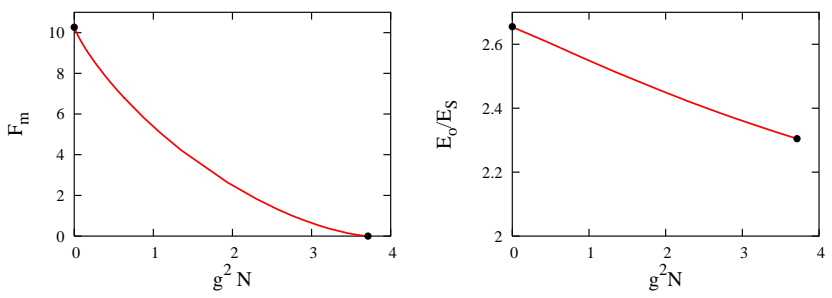

FIG. 1. Suppression exponent (left) and energy of realtime instantons (right) versus the number of incoming particles in the model (17).

number of particles it is convenient to recast Eq. (14) in the form

$$
\tilde{N} \equiv g^{2} N=-\frac{2}{\operatorname{sh} \theta} \int d \xi a^{\prime}(\xi) b(\xi) .
$$

Functions $F_{\mathrm{m}}(\theta), E_{\mathrm{o}}(\theta)$ and $\tilde{N}(\theta)$ determine the dependence of the suppression exponent and the energy on $\tilde{N}$. These are plotted in Fig. 1 Taking the limit $\tilde{N} \rightarrow 0$ one obtains $F_{\mathrm{m}}=10.27, E_{\mathrm{o}}=2.65 E_{S}$. Thus, the semiclassical suppression factor is $\exp \left(-10.27 / g^{2}\right)$ at all energies exceeding $E_{\mathrm{o}}=2.65 E_{S}$, and the suppression is even stronger at lower energies.

In conclusion we summarize our method. To calculate the minimum suppression exponent $F_{\mathrm{m}}$ and the optimal energy $E_{\mathrm{o}}$ of a collision-induced tunneling process, one finds the family of complex classical solutions, real-time instantons, satisfying the boundary conditions (13). Given the real-time instantons, one calculates the number of particles $\tilde{N}$, the suppression exponent $F_{\mathrm{m}}(\tilde{N})$, Eqs. (14), (15), and the energy $E_{\mathrm{o}}(\tilde{N})$. By itself, the quantity $F_{\mathrm{m}}(\tilde{N})$ provides the lower bound on $F_{\mathrm{m}}$, Eq. (5); the limit $\tilde{N} \rightarrow 0$ yields $F_{\mathrm{m}}$ and $E_{\mathrm{o}}$ according to Eqs. (6), (16).

We are indebted to S. Dubovsky, V. Rubakov, P. Tinyakov and F. Bezrukov for helpful suggestions. This work has been supported in part by RFBR grant 02-02-17398, grant NS-2184.2003.2 and fellowships of the "Dynasty" foundation (awarded by the Scientific board of ICFPM). Work of D. L. has been supported by CRDF award RP1-2364-MO-02 and INTAS grant YS03-55-2362. D.L. is grateful to the Universite Libre de Bruxelles for hospitality.

1. S. R. Coleman, Phys. Rev. D 15, 2929 (1977) [Erratumibid. D 16, 1248 (1977)]; HUTP-78/A004 Lecture delivered at 1977 Int. School of Subnuclear Physics, Erice, Italy, Jul 23-Aug 10, 1977

2. A. A. Belavin, A. M. Polyakov, A. S. Schwartz and Y. S. Tyupkin, Phys. Lett. B 59, 85 (1975).
3. N. S. Manton, Phys. Rev. D 28, 2019 (1983). F. R. Klinkhamer and N. S. Manton, Phys. Rev. D30, 2212 (1984).

4. A. Ringwald, Nucl. Phys. B330, 1 (1990). O. Espinosa, Nucl. Phys. B343, 310 (1990).

5. A. N. Kuznetsov and P. G. Tinyakov, Phys. Rev. D56, 1156 (1997).

6. F. Bezrukov, D. Levkov, C. Rebbi, V. Rubakov and P. Tinyakov, Phys. Rev. D 68, 036005 (2003); Phys. Lett. B 574, 75 (2003).

7. M. B. Voloshin, Phys. Rev. D 49, 2014 (1994).

8. V. A. Rubakov and D. T. Son, Nucl. Phys. B 424, 55 (1994).

9. V. I. Zakharov, Nucl. Phys. B 353, 683 (1991).

G. Veneziano, Mod. Phys. Lett. A 7, 1661 (1992).

M. Maggiore and M. A. Shifman, Nucl. Phys. B 371, 177 (1992).

10. D. G. Levkov and S. M. Sibiryakov, arXiv:hep-th/0410198

11. V. A. Rubakov, D. T. Son and P. G. Tinyakov, Phys. Lett. B 287, 342 (1992).

12. P. G. Tinyakov, Phys. Lett. B 284, 410 (1992). A. H. Mueller, Nucl. Phys. B 401, 93 (1993).

13. G. F. Bonini, A. G. Cohen, C. Rebbi and V. A. Rubakov, Phys. Rev. D 60, 076004 (1999).

14. F. Bezrukov and D. Levkov, J. Exp. Theor. Phys. 98, 820 (2004) [Zh. Eksp. Teor. Fiz. 125, 938 (2004)].

15. P. G. Tinyakov, Int. J. Mod. Phys. A 8 (1993) 1823.

16. C. Rebbi and R. J. Singleton, hep-ph/9706424

17. C. L. Kane and M. P. A. Fisher, Phys. Rev. Lett. 68, 1220 (1992).

18. R. Fazio, K. H. Wagenblasta, C. Winkelholza and G. Schön, Physica B222, 364 (1996). 